\title{
Experiencia de cinco años en el manejo de pacientes con alto riesgo de coledocolitiasis
}

\author{
5 -year experience in the management of patients \\ with high risk of choledocholithiasis
}

\author{
José de Jesús Marín-López, ${ }^{*}$ Efrén Flores-Álvarez, ${ }^{\ddagger}$ Ramiro Gómez-Arámbulo, ${ }^{\S}$ José Cruz De la Torre Gonzáles, ${ }^{\S}$ \\ Danyel Chávez Fernández, ${ }^{*}$ José Augusto Rodríguez Osuna, ${ }^{*}$ Josué Israel Olivares del Moral§ \\ * Especialidad en Cirugía General. ₹ Posgrado en Cirugía General. \\ $\S$ Departamento de Cirugía General. \\ Centenario Hospital «Miguel Hidalgo». Aguascalientes, Ags.
}

\section{RESUMEN}

Introducción: La coledocolitiasis se desarrolla entre 8 y $18 \%$ de los pacientes con colecistitis litiásica. El diagnóstico se establece con base en los criterios de la American Society for Gastrointestinal Endoscopy (ASGE). En pacientes con alto riesgo, el tratamiento sugerido es colangiopancreatografía retrógrada endoscópica (CPRE). Aunque la exploración quirúrgica de la vía biliar (EVB) ha demostrado resultados similares en cuanto a efectividad y complicaciones, aún existe controversia respecto a la mejor opción de tratamiento. Objetivo: Análisis y descripción de nuestra experiencia en el manejo de pacientes con alto riesgo de coledocolitiasis en el Centenario Hospital Miguel Hidalgo en un periodo de cinco años. Material y métodos: Estudio tipo serie de casos, retrospectivo, descriptivo, analítico y comparativo de pacientes con alto riesgo de coledocolitiasis, generando dos grupos con base en el tratamiento: colangiopancreatografía retrógrada endoscópica (CPRE) o exploración de vía biliar (EVB). Descripción de variables cualitativas y cuantitativas con porcentajes y medidas de tendencia central, $\chi^{2}$ para variables categóricas, t de Student o U Mann-Whitney, según normalidad de los datos. Resultados: Muestra de 76 pacientes con alto riesgo de coledocolitiasis, $55(72.37 \%)$ mujeres y $21(27.63 \%)$ hombres. Media de edad 43 años ( $D E \pm 19.07)$. La CPRE fue terapéutica en 41 casos $(56.94 \%$ ), no terapéutica en 31 (vía biliar no permeable, $n=20$; ausencia

\section{ABSTRACT}

Introduction: Choledocholithiasis develops in 8 to $18 \%$ of patients with lithiasic cholecystitis. The diagnosis is established based on the criteria of the American Society for Gastrointestinal Endoscopy (ASGE). In high-risk patients, the suggested treatment is endoscopic retrograde cholangiopancreatography (ERCP), although the common bile duct exploration (BDE) has shown similar results in terms of effectiveness and complications. There is still controversy regarding the best treatment option. Objective: Analysis and description of our experience in the management of patients with high risk of choledocholithiasis in the Centenario Hospital Miguel Hidalgo in a period of five years. Material and methods: Retrospective, descriptive, analytical and comparative case series study of patients with high risk of choledocholithiasis, generating two groups based on treatment: endoscopic retrograde cholangiopancreatography (ERCP) or bile duct exploration (BDE). Description of qualitative and quantitative variables with percentages and measures of central tendency, $\chi^{2}$ for categorical variables, Student t or U Mann-Whitney according to data normality. Results: Sample of 76 patients with high risk of choledocholithiasis, 55 (72.37\%) female and 21 (27.63\%) male. Mean age 43 years $(S D \pm 19.07)$. ERCP was therapeutic in 41 cases (56.94\%), non-therapeutic in 31 (no bile duct clearance, $n=$ 20; stone absence, $n=11$ ) and ERCP was not performed in four cases.

Recibido para publicación: 10/01/2020. Aceptado: 03/07/2020.

Correspondencia: José de Jesús Marín-López. Residente de Cirugía General. Av. Gómez Morín s/n, Col. La Estación, Alameda, 20259, Aguascalientes, Aguascalientes, México. Teléfono: 44 9414-2361. E-mail: jesua_987@hotmail.com

Citar como: Marín-López JJ, Flores-Álvarez E, Gómez-Arámbulo R, De la Torre GJC, Chávez FD, Rodríguez OJA et al.

Experiencia de cinco años en el manejo de pacientes con alto riesgo de coledocolitiasis. Rev Mex Cir Endoscop. 2020; 21 (1): 26-31. https://dx.doi.org/10.35366/97610 
de litos, $n=11$ ) y no se realizó CPRE en cuatro casos. Se trataron 24 pacientes con EVB, resolviéndose $95 \%$. Sin asociación entre tipo de abordaje y complicaciones $(p=0.823)$. La pancreatitis pos-CPRE se encontró en $18.42 \%$. Estancia hospitalaria con CPRE fue de nueve días $( \pm 12,2-87)$ y con EVB de ocho días $( \pm 5,2-21)$. Conclusión: El procedimiento con más casos resueltos en nuestro medio es la EVB. Decidir la estrategia de tratamiento dependerá de la experiencia e insumos en cada institución.

Palabras clave: Coledocolitiasis, CPRE, pancreatitis pos-CPRE, exploración de vía biliar.
24 patients were treated with $B D E$, resolving $95 \%$. No association found between type of approach and complications ( $p=0.823)$. Pos-ERCP pancreatitis occurs in $18.42 \%$. Hospital stay with ERCP was 9 days $( \pm$ $12,2-87)$ and with $E V B 8$ days ( $\pm 5,2-21)$. Conclusion: In our sample the $B D E$ is the procedure with most cases resolved. Deciding the treatment strategy will depend of the experience and resource in each institution.

Keywords: Choledocholithiasis, ERCP, post-ERCP pancreatitis, bile duct exploration.

\section{INTRODUCCIÓN}

La coledocolitiasis $(\mathrm{CL})$ es un padecimiento con una definición puntual y sencilla: «Presencia de litos en la vía biliar principal». Se clasifica, según la etiología del lito como primaria (el proceso de litogénesis se origina dentro de la vía biliar como consecuencia de estasis por etiologías múltiples) o secundaria (el lito migra desde la vesícula biliar, pasa a través del cístico y termina en la vía biliar principal). Esta última es la forma de presentación en 85\% de los casos. ${ }^{1-4}$ La prevalencia de coledocolitiasis en los pacientes con colecistitis litiásica que se reporta en las guías ASGE y ESGE es de 8 a 18\%.5,6 En México, la proporción es de 5 a 10\%. ${ }^{7}$ La probabilidad del diagnóstico se establecía hasta hace algunos meses con base en los criterios de la American Society for Gastrointestinal Endoscopy (ASGE) 2010, los cuales fueron modificados en junio del año 2019 con la finalidad de mejorar su sensibilidad y especificidad, retirando el diagnóstico de pancreatitis aguda como predictor de coledocolitiasis. ${ }^{8,9}$ Sin embargo, ambas guías estratifican la probabilidad de coledocolitiasis en tres grupos (riesgo alto, intermedio y bajo) de acuerdo con datos clínicos, resultados de estudios de laboratorio e imagen sin haberse modificado los parámetros que definen alto riesgo de coledocolitiasis. ${ }^{10}$ Para los pacientes con alto riesgo el tratamiento inicial sugerido es la colangiopancreatografía retrógrada endoscópica (CPRE), aunque la exploración quirúrgica de la vía biliar (EVB) ha demostrado resultados similares en cuanto a complicaciones y efectividad para la extracción de litos (93.3 vs $97.7 \%$, respectivamente), por lo tanto, aún existe controversia respecto a cuál es la mejor opción de tratamiento y en qué escenario. ${ }^{11-13}$ Cabe resaltar que todas las guías, ensayos clínicos y metaanálisis al respecto pautan, a pesar de la evidencia, que el manejo definitivo del paciente con coledocolitiasis debe adaptarse a los recursos disponibles para su manejo en cada institución. ${ }^{14-18}$ La nuestra tiene un servicio de endoscopía que dispone de guía hidrofílica, canastilla de Dormia, balones y endoprótesis plástica, fluoroscopio y medio de contraste sin tener acceso a SpyGlass, coledocoscopios o litotriptores endoscópicos. Para una resolución quirúrgica, contamos con equipo de exploración para vías biliares laparoscópico y con material para exploración abierta; colangiografía transoperatoria y broncoscopio o cistoscopio para revisión del colédoco. El objetivo del estudio es la descripción de nuestra experiencia en el manejo de pacientes con alto riesgo de coledocolitiasis en el Centenario Hospital Miguel Hidalgo en un periodo de cinco años.

\section{MATERIAL Y MÉTODOS}

Se realizó un estudio que comprendió un periodo de cinco años (enero de 2011 a enero de 2016); tipo serie de casos, retrospectivo, descriptivo, analítico y comparativo. La selección de casos que componen la muestra se realizó a través de la revisión de expedientes clínicos del archivo hospitalario, incluyendo a pacientes con diagnóstico de coledocolitiasis, según los criterios de la ASGE 2010, tomando para fines de este estudio sólo aquéllos con alto riesgo y realizando una descripción de los parámetros de laboratorio (pruebas de funcionamiento hepático) e imagen (ultrasonido transabdominal de hígado y vías biliares) asociados al diagnóstico, así como un análisis del tratamiento implementado, ya fuera

\section{Tabla 1: Parámetros de laboratorio de la muestra.}

Variable

$n=76$

GGT UI/L (media \pm DE)

Fosfatasa alcalina UI/L (media $\pm \mathrm{DE}$ )

AST UI/L (media $\pm D E)$

ALT UI/L (media $\pm D E)$

Bilirrubina total $\mathrm{mg} / \mathrm{dL}$ (media $\pm \mathrm{DE}$ )

Bilirrubina directa $\mathrm{mg} / \mathrm{dL}$ (media $\pm \mathrm{DE}$ )

Diámetro colédoco mm (media $\pm \mathrm{DE}$ )

Lito visible en USG ( $\mathrm{n} / \%)$

- Diámetro del lito

$582 \pm 406.4(160-2,124)$

$283 \pm 177.5(44-980)$

$237 \pm 211.1(16-915)$

$279 \pm 201(16-854)$

$6.1 \pm 6.3(0.67-42)$

$4.7 \pm 4.8(0.30-31.4)$

$10.6 \pm 4.7(3-25)$

$23(30.2 \%)$

$13.4 \pm 6.7(5-27)$

GGT = gamma-glutamil transpeptidasa, AST = aspartato aminotransferasa, ALT = alanina aminotransferasa, USG = ultrasonido transabdominal. 


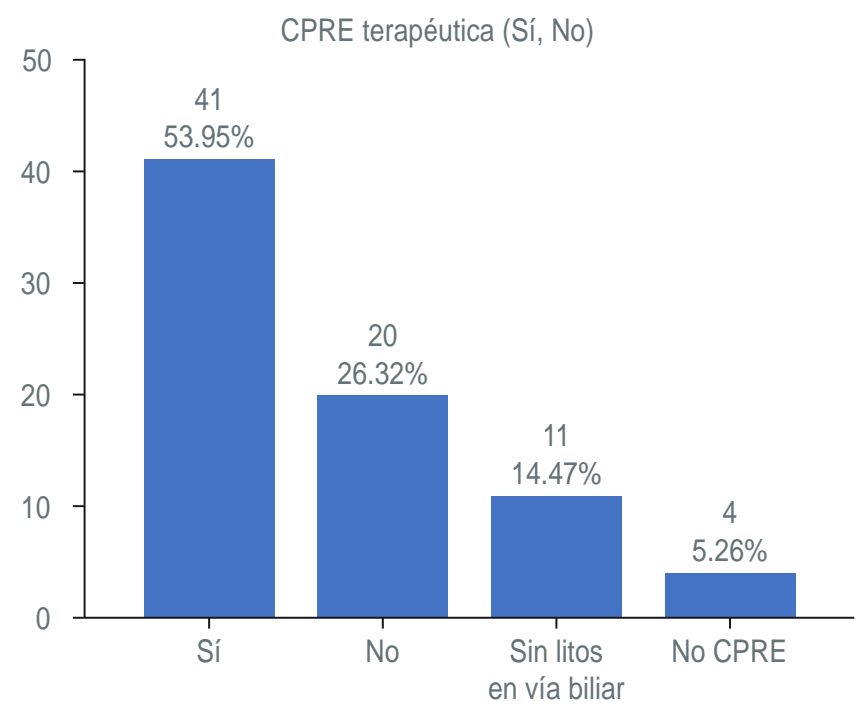

Figura 1: Pacientes con alto riesgo de coledocolitiasis divididos acorde a los hallazgos de la colangiopancreatografía retrógrada endoscópica (CPRE).

endoscópico (CPRE), exploración de vía biliar (EVB) laparoscópica o abierta y variables asociadas como porcentaje de casos en los que se permeabiliza la vía biliar, tiempo quirúrgico, días de estancia hospitalaria y complicaciones asociadas. Para la descripción de variables cualitativas y cuantitativas se usaron porcentajes y medidas de tendencia central, así como prueba de $\chi^{2}$ para buscar asociación entre variables categóricas y prueba t de Student o U Mann-Whitney, según normalidad de los datos. El análisis estadístico se llevó a cabo con el software de IBM ${ }^{\odot}$ SPSS $^{\odot}$ Statistic V21.

\section{RESULTADOS}

Se identificaron 76 pacientes con diagnóstico de alto riesgo de coledocolitiasis en dicho periodo, de los cuales $55(72.37 \%)$ fueron del sexo femenino y 21 (27.63\%) del masculino. Presentaron una media de edad de 43 años (DE $\pm 19.07,16-86)$. Respecto a los parámetros de laboratorio e imagen de la muestra concernientes al diagnóstico (Tabla 1), la media de bilirrubina total fue de $6.1 \mathrm{mg} / \mathrm{dL}$ ( $\mathrm{DE} \pm$ 6.3, 0.67-42), bilirrubina directa de $4.7 \mathrm{mg} / \mathrm{dL}$ (DE \pm 4.8 , $0.30-31.4)$ y la media del diámetro del colédoco fue de $10.6 \mathrm{~mm}$ ( $\mathrm{DE} \pm 4.7,3-25)$, con litos visibles por ultrasonido (USG) dentro del colédoco en 23 casos (30.26\%), los cuales presentaron una media de $13.4 \mathrm{~mm}$ de diámetro ( $\mathrm{DE} \pm 6.7,5-27$ ). Todos se trataron inicialmente de forma endoscópica, a excepción de cuatro casos que se llevaron directamente a EVB por criterio del cirujano tratante. El resto $(n=72,94.7 \%)$ se dividió en función de los resultados de la CPRE en Terapéutica o No terapéutica. De acuerdo con este criterio se identificaron 41 casos (56.94\%) en que fue terapéutica, es decir, extracción exitosa de litos y permeabilidad completa de la vía biliar. En 11 pacientes no se encontraron litos al momento de realizar la CPRE y en 20 no se logró extraer los litos de la vía biliar, considerándose ambos como no terapéutica ( $n=31,43.05 \%)$. Estos últimos casos en los que no se logró extraer los litos se sometieron a exploración de vía biliar (EVB), al igual que los cuatro pacientes en los que no se realizó CPRE de manera inicial $(n=24)$, resumiéndose las variables de este grupo en la Tabla 2. El abordaje quirúrgico se realizó de acuerdo con la disponibilidad de insumos y experiencia del cirujano de la siguiente manera: Exploración abierta en siete pacientes (29.17\%) y exploración laparoscópica en 17 (70.83\%). El tipo de manejo durante la exploración se resume en la Tabla 3 , donde se aprecia un caso de litos residuales que no fue advertido durante la colangiografía transoperatoria a través del cístico, el cual generó sintomatología en el posoperatorio y se resolvió con endoscopía. El cierre primario del colédoco se realizó en 18 casos (74.9\%); 13 fueron sobre una prótesis plástica colocada previamente por endoscopía y en cinco, cierre sin prótesis. En tres el cierre del colédoco fue sobre una sonda Kehr. El material de sutura empleado fue poliglactina 3-0 en 14 casos y PDS 3-0 en siete, con cierre continuo en un paciente y puntos separados en 20 (95.24\%). Al contraste de variables entre un procedimiento y otro (Tabla 4) encontramos que respecto al tiempo quirúrgico existe diferencia significativa a favor del abordaje endoscópico ( $p=0.000)$; en días de estancia hospitalaria no existe diferencia estadísticamente significativa $(p=0.479)$ y analizando exclusivamente la estancia de los pacientes que se llevaron directamente a EVB, encontramos tres casos con cinco días y uno con nueve días. En complicaciones, el abordaje endoscópico presentó una prevalencia de pancreatitis pos-CPRE de 18.4\% $(n=14)$, con un caso de perforación duodenal Stapfer IV, tratada con vigilancia y ayuno. En la EVB presentaron infección de sitio quirúrgico $(n=2)$, colección intraabdominal secundaria a fuga biliar más sepsis abdominal $(n=1)$, litos residuales $(n=1)$. No

\section{Tabla 2: Variables de los casos sometidos a EVB}

Variable

Bilirrubina total (media $\pm \mathrm{DE})$

Bilirrubina directa (media $\pm \mathrm{DE})$

Colédoco $(\mathrm{mm})($ media $\pm \mathrm{DE})$

Lito visible en USG ( $\mathrm{n} / \%)$

- Diámetro del lito $\geq$ a $20 \mathrm{~mm}$ $n=24$

$9.1 \pm 9.9(0.67-42)$

$7.1 \pm 7.2(0.41-319$

$13.9 \pm 5.6(6-25)$

$11(45.8)$

6
EVB = exploración de vía biliar, USG = ultrasonido transabdominal. 


\begin{tabular}{|c|c|c|}
\hline $\begin{array}{c}\text { EVB } \\
n=24\end{array}$ & $\begin{array}{l}\text { Abordaje } \\
\text { abierto } \\
(n=7)\end{array}$ & $\begin{array}{c}\text { Abordaje } \\
\text { laparoscópico } \\
(n=17)\end{array}$ \\
\hline \multicolumn{3}{|l|}{ Vía de acceso } \\
\hline $\begin{array}{l}\text { ClO transcística + extracción } \\
\text { transcística de litos }\end{array}$ & 2 & 1 \\
\hline $\begin{array}{l}\text { Coledocotomía + coledocos- } \\
\text { copía* + extracción de litos }\end{array}$ & 5 & 16 \\
\hline \multicolumn{3}{|l|}{ Tipo de cierre } \\
\hline CP sobre EP & 2 & 11 \\
\hline $\mathrm{CP}$ & 1 & 4 \\
\hline Sonda T-cístico & 1 & 0 \\
\hline Sonda T-colédoco & 2 & 1 \\
\hline Vía biliar permeable & 7 & 16 \\
\hline
\end{tabular}

$\mathrm{CIO}=$ colangiografía intraoperatoria, $\mathrm{CP}=$ cierre primario, $\mathrm{EP}=$ endoprótesis.

*Endoscopio $8.5 \mathrm{~mm}$, cistoscopio y broncoscopio según disponibilidad.

se encontró asociación entre el tipo de procedimiento, CPRE o EVB, con relación a complicaciones $(p=0.823)$. Se confirmó la presencia de litos dentro de vía biliar en $85.5 \%$ de la muestra y la EVB resolvió $95.8 \%(n=23)$ de los casos de coledocolitiasis y la CPRE 56.94\% $(n=41)$.

\section{DISCUSIÓN}

Los hallazgos en este estudio muestran que la mitad de los casos con alto riesgo de coledocolitiasis se resuelven con CPRE (56.4\%), lo cual contrasta con el éxito terapéutico de 85 a 90\% reportado en otras series con el primer procedimiento endoscópico. ${ }^{19,20}$ Este resultado puede deberse al número de procedimientos que no fueron terapéuticos como consecuencia de dos eventos: Ausencia de litos durante la CPRE o no lograr su extracción de la vía biliar. Esto podría ser porque los criterios para coledocolitiasis de la ASGE en la población mexicana, según Narváez y colaboradores, tienen baja sensibilidad y especificidad con valor predictivo positivo de 13 a 58\% para alto riesgo de coledocolitiasis. ${ }^{21}$ Sólo de 10 a 60\% tendrán litos, lo cual generaría de 40 a $50 \%$ de CPRE innecesarias y por tanto, no terapéuticas. ${ }^{22-24} \mathrm{Sin}$ embargo, en $85.5 \%$ de la muestra se confirmó la presencia de litos. Podemos apreciar que la media de las variables: Bilirrubina total, diámetro del colédoco y diámetro de los litos es mayor en los casos en los que la CPRE no logró la extracción de litos con respecto a aquéllos en los que fue terapéutica. Algunos autores mencionan que cuando fallan los métodos convencionales de extracción, se considera como «lito difícil» y para resolverlo es necesario equipo endoscópico complementario. ${ }^{25} \mathrm{Si}$ bien no existe consenso respecto a la definición de este concepto, Üsküdar y colaboradores proponen una definición aplicable a nuestros casos. ${ }^{26}$ Estos pacientes en los que la CPRE no logró la extracción de litos, fueron tratados con EVB, que independiente del tipo de abordaje, presentó un porcentaje mayor de casos resueltos. Esto puede atribuirse a la experiencia y disponibilidad de insumos para tal procedimiento. Respecto a la media de estancia hospitalaria de nuestra serie, encontramos que es mayor con relación a lo reportado en la literatura de $4.6 \pm 4.1$ días. $^{27,28}$ Lo anterior, porque el abordaje inicial en toda la muestra fue endoscópico y posteriormente quirúrgico para los casos mencionados, y por las comorbilidades que se presentaron, siendo la más frecuente pancreatitis pos-CPRE (18.4\%), que asociamos al tipo de instrumentación como esfinterotomía y dilatación con balón. Bajo estas condiciones, la literatura refiere de 15.4 a $20 \%$ de pancreatitis pos-CPRE. ${ }^{29,30}$ En cuanto a la EVB la complicación que ameritó mayor estancia hospitalaria para su tratamiento fue un caso de fuga biliar más sepsis abdominal que se presentó en el grupo de abordaje laparoscópico con sonda Kehr en el colédoco. Finalmente, todos los pacientes con alto riesgo de coledocolitiasis de esta muestra se resolvieron, aunque debemos mejorar el tiempo de estancia hospitalaria y comorbilidades a partir de diseñar una estrategia de tratamiento que considere estudios de imagen como la colangiopancreatografía por resonancia magnética (CPRM), tal como Chander y colaboradores. ${ }^{31-34}$ Esto además de confirmar el diagnóstico de alto riesgo de coledocolitiasis, proporcionaría datos respecto a la anatomía de la vía biliar, su calibre, diámetro y cantidad de litos, permitiendo definir y ajustar el tratamiento de

Tabla 4: Comparación entre CPRE y EVB.

\begin{tabular}{|c|c|c|c|}
\hline Variable & $\begin{array}{c}\text { CPRE } \\
(n=52)\end{array}$ & $\begin{array}{c}\text { EVB } \\
(n=24)\end{array}$ & $\begin{array}{l}p= \\
0.05\end{array}$ \\
\hline $\begin{array}{l}\text { Tiempo quirúrgico } \\
\text { (min) }\end{array}$ & $\begin{array}{c}73.4( \pm 52.5 \\
35-300)\end{array}$ & $\begin{array}{c}210( \pm 77.5 \\
90-341)\end{array}$ & 0.000 \\
\hline $\begin{array}{l}\text { Estancia hospital } \\
\text { (días) }\end{array}$ & $\begin{array}{c}9( \pm 12 \\
2-87)\end{array}$ & $\begin{array}{l}8( \pm 5 \\
2-21)\end{array}$ & 0.479 \\
\hline \multicolumn{4}{|l|}{$\begin{array}{l}\text { Complicaciones } \\
\text { n (\%) }\end{array}$} \\
\hline Sí & $15(28.8)$ & $4(16.6)$ & - \\
\hline No & $37(71.1)$ & $20(83.3)$ & - \\
\hline $\begin{array}{l}\text { Lito confirmado en } \\
\text { vía biliar (n) }\end{array}$ & 41 & 24 & - \\
\hline Casos resueltos (\%) & 56.94 & 95.8 & - \\
\hline
\end{tabular}

$\mathrm{CPRE}=$ colangiopancreatografía retrógrada endoscópica, $\mathrm{EVB}=$ exploración de vía biliar. 
acuerdo con los recursos de la unidad tratante con relación a la experiencia del cirujano o endoscopista. Este análisis orienta a que el procedimiento con más casos resueltos en nuestro medio es la EVB. Sin embargo, para determinarlo con una evidencia fuerte, es necesario realizar un ensayo clínico que compare ambos tratamientos en igualdad de condiciones. Por lo tanto, los resultados de esta muestra no son suficientes para realizar inferencias confiables respecto a qué abordaje terapéutico es mejor.

\section{CONCLUSIONES}

El objetivo principal con los pacientes que presentan alto riesgo de coledocolitiasis es la extracción de litos y la permeabilización de la vía biliar, por lo que decidir la estrategia de tratamiento es crucial y dependerá de la experiencia e insumos en cada institución. En la nuestra, todos los casos con alto riesgo de coledocolitiasis son tratados inicialmente con CPRE, y aquéllos no resueltos son llevados a exploración quirúrgica de vía biliar, siendo esta última el medio que con mayor frecuencia logra permeabilizar la vía biliar.

\section{REFERENCIAS}

1. Bolívar-Rodríguez MA, Pamanes-Lozano A, Corona-Sapien CF et al. Coledocolitiasis. Una revisión. Rev Med UAS. 2017; 7: 138-154.

2. González-Pérez L, Zaldívar-Ramírez FR, Tapia-Contl BR et al. Factores de riesgo de la coledocolitiasis asintomática; experiencia en el Hospital General de México. Cir Gen. 2018; 40: 164-168.

3. Tejedor-Bravo M, Albillos-Martínez A. Enfermedad litiásica Biliar. Medicine. 2012; 11: 481-488.

4. Zorrilla CF. Coledocolitiasis primaria y secundaria y su resolución. En: Morales Saavedra JL. Tratado de cirugía general. 3a ed. México: Manual Moderno; 2017. pp. 1350-1354.

5. Manes G, Paspatis G, Aabakken L et al. Endoscopic management of common bile duct stones: European Society of Gastrointestinal Endoscopy (ESGE) guideline. Endoscopy. 2019; 51: 472-491.

6. Cuendis-Velázquez A, Rojano-Rodríguez ME, MoralesChávez CE et al. Utilidad de la coledocoscopía transquirúrgica en el tratamiento de litos biliares difíciles. Rev Gastroenterol Méx. 2014; 79: 22-27.

7. Tazuma S, Unno M, Igarashi Y et al. Evidence-based clinical practice guidelines for cholelithiasis 2016. J Gastroenterol. 2017; 52: 276-300.

8. ASGE Standards of Practice Committee, Maple JT, BenMenachem T, Anderson MA et al. The role of endoscopy in the evaluation of suspected choledocholithiasis. Gastrointest Endosc. 2010; 71: 1-9.

9. ASGE Standards of Practice Committee, Maple JT, BenMenachem T, Anderson MA et al. The role of endoscopy in the evaluation and management of choledocholithiasis. Gastrointest Endosc. 2019; 89: 1075-1105.
10. Sousa M, et al. ASGE high-risk criteria for choledocholithiasisAre they applicable in cholecystectomized patients? Dig Liver Dis. 2018;51(1):75-78.

11. Taylor EW, Rajgopal U, Festekjian J. The efficacy of preoperative endoscopic retrograde cholangiopancreatography in the detection and clearance of choledocholithiasis. JSLS. 2000; 4: 109-116.

12. Gad EH, Zakaria H, Kamel Y et al. Surgical (Open and laparoscopic) management of large difficult CBD stones after different sessions of endoscopic failure: A retrospective cohort study. Ann Med Surg (Lond). 2019; 43: 52-63.

13. Gao YC, Chen J, Qin Q et al. Efficacy and safety of laparoscopic bile duct exploration versus endoscopic sphincterotomy for concomitant gallstones and common bile duct stones: a meta-analysis of randomized controlled trials. Medicine (Baltimore). 2017; 96: e7925.

14. Aleknaite A, Simutis G, Stanaitis J et al. Risk assessment of choledocholithiasis prior to laparoscopic cholecystectomy and its management options. United European Gastroenterol J. 2018; 6: 428-438.

15. Dasari BV, Tan CJ, Gurusamy KS, Martin DJ, Kirk G, McKie $L$ et al. Surgical versus endoscopic treatment of bile duct stones. Cochrane Database Syst Rev. 2013; (9): CD003327.

16. Chiappetta PL, Canullan C. Tratamiento laparoscópico de la coledocolitiasis. En: Galindo F. Cirugía digestiva. www. sacd.org.ar, 2009; IV-451, pp. 1-9.

17. Williams E, Beckingham I, El Sayed G et al. Updated guideline on the management of common bile duct stones. Gut. 2017; 66: 765-782.

18. Narula VK, Fung EC, Overby DW, Richardson W, Dimitrios Stefanidis and the SAGES Guidelines Committee. Clinical spotlight review: management of choledocholithiasis. 2020. Available in: sages.org/publications/ guidelines/clinical-spotlight-review-management-ofcholedocholithiasis.

19. Trikudanathan G, Navaneethan U, Parsi MA. Endoscopic management of difficult common bile duct stones. World J Gastroenterol. 2013; 19: 165-173.

20. Aburajab M, Dua K. Endoscopic management of difficult bile duct stones. Curr Gastroenterol Rep. 2018; 20 (2): 8.

21. Nárvaez Rivera RM, González González JA, Monreal Robles R, García Compean D, Paz Delgadillo J, Garza Galindo AA et al. Accuracy of ASGE criteria for the prediction of choledocholithiasis. Rev Esp Enferm Dig. 2016; 108: 309-314.

22. Ripari G, Wulfson A, Guerrina C, Perroud H. Correlación entre predictores de litiasis coledociana y los hallazgos en la colangiopancreatografía retrógrada endoscópica. De las guías a la práctica. Acta Gastroenterol Latinoam. 2017; 47: 269-276.

23. Costi R, Gnocchi A, Di Mario F, Sarli L. Diagnosis and management of choledocholithiasis in the golden age of imaging, endoscopy and laparoscopy. World J Gastroenterol. 2014; 20: 13382-13401.

24. Barreras González JE, Torres Peña R, Ruiz Torres J, Martínez Alfonso MÁ, Brizuela Quintanilla R, Morera 
Pérez M. Endoscopic versus laparoscopic treatment for choledocholithiasis: a prospective randomized controlled trial. Endosc Int Open. 2016; 4: E1188-E1193.

25. Doshi B, Yasuda I, Ryozawa S, Lee GH. Current endoscopic strategies for managing large bile duct stones. Dig Endosc. 2018; 30 Suppl 1: 59-66.

26. Üsküdar O, Parlak E, Dışıbeyaz S, Köksal AS, Cıçek B, Kılıç ZM et al. Major predictors for difficult common bile duct stone. Turk J Gastroenterol. 2013; 24: 423-429.

27. Magalhães J, Rosa B, Cotter J. Endoscopic retrograde cholangiopancreatography for suspected choledocholithiasis: from guidelines to clinical practice. World J Gastrointest Endosc. 2015; 7: 128-134.

28. Sharma A, Dahiya P, Khullar R, Soni V, Baijal M, Chowbey PK. Management of common bile duct stones in the laparoscopic era. Indian J Surg. 2012; 74: 264-269.

29. Katsinelos P, Lazaraki G, Chatzimavroudis G, Gkagkalis S, Vasiliadis I, Papaeuthimiou A et al. Risk factors for therapeutic ERCP-related complications: an analysis of 2,715 cases performed by a single endoscopist. Ann Gastroenterol. 2014; 27: 65-72.

30. Kwon $\mathrm{Cl}$, Song $\mathrm{SH}$, Hahm KB, Ko KH. Unusual complications related to endoscopic retrograde cholangiopancreatography and its endoscopic treatment. Clin Endosc. 2013; 46: 251-259.

31. Chander J, Vindal A, Lal P, Gupta N, Ramteke VK. Laparoscopic management of CBD stones: an Indian experience. Surg Endosc. 2011; 25: 172-181.

32. Senturk S, Miroglu TC, Bilici A, Gumus H, Tekin RC, Ekici $\mathrm{F}$ et al. Diameters of the common bile duct in adults and postcholecystectomy patients: a study with 64-slice CT. Eur J Radiol. 2012; 81: 39-42.

33. Horrow MM. Ultrasound of the extrahepatic bile duct: issues of size. Ultrasound Q. 2010; 26: 67-74.

34. Gómez-Zuleta M, Ruiz-Morales O, Otero-Regino W. What is the normal size of the common bile duct? Rev Colomb Gastroenterol. 2017; 32 (2): 96-104. 